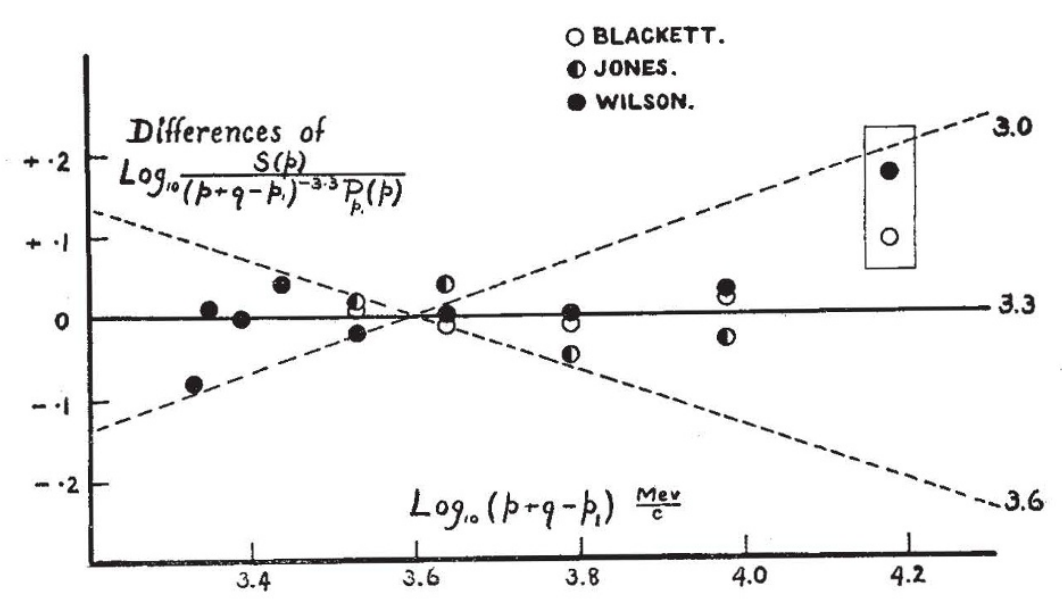

$S_{0}(p) \sim p^{-3 \cdot 3}$

(5)

we find (4) to be reasonably satisfied. To show the agreement we have plotted in the accompanying illustration differences of

$$
\log \left\{\frac{S(p)}{\left(p+q-p_{1}\right)^{-3.3} P_{p_{1}}(p)}\right\}
$$

from an arbitrary zero against

$$
\log \left\{p+q-p_{1}\right\}
$$

The observed points lie reasonably well on a horizontal line, supporting equation (5).

We indicate by broken lines in the illustration the changes of slope in the scale of the diagram, which correspond to exponents $3 \cdot 0$ and 3.6 in place of $3.3 ;$ the experimental points are clearly inconsistent with so large a variation from the latter flgure.

The above analysis is very similar to that of Euler and Heisenberg, which leads to an exponent $2 \cdot 87$ instead of the exponent $3 \cdot 3$ we find here. The present value is based on much more extensive observational material and is, therefore, probably the more reliable value.

An appreciable deviation is noticeable in the diagram for the point at $\log _{10}\left(p+q-p_{1}\right)=4 \cdot 18$. This undoubtedly oceurs because at this momentum the limit of resolution of the measuring equipment is approached. It was flrst pointed out by Blackett that at momenta greater than this limit the apparent measured differential spectrum is determined solely by errors of measurement and must be of exponent -2. The deviation which occurs is in accord with the observed resolution of the spectra concerned.

L. JANOSSY

Physical Laboratories,
University of Manchester. Aug. 16.

1 Euler and Heisenberg, Ergeb. exakt. Naturwiss., 17, 1 (1938). ${ }^{2}$ Nature, 158, 414 (1946).

\section{Thermal Migration of Macromolecules}

DEBYE has reported ${ }^{1}$ that the velocity of thermal diffusion, relative to that of ordinary diffusion, is much greater in polymer solutions than in simple solutions. This result is likely to arouse a great deal of interest, and the following recent observations of our own concerning thermal diffusion in solutions of polymethyl methacrylate in various solvents may therefore be opportune.

Unlike Debye and his co-workers, we have not found it necessary, in order to demonstrate the effect, to use a method as elaborate as that of Clusius and Dickel. The process is so rapid, and the concentration gradient so great, that the reduction of viscosity in the region of high temperature is often more than compensated for by the local high temperature is often more than compensated for by the local
increase in concentration of the polymer solution. A very simple increase in concentration of the polymer solution. A very simple beaker containing a cold solution of the polymer in toluene on a hot surface and then, a few seconds later, to detect the formation of a treacly layer at the bottom by careful scraping with a glass rod, or by watching adventitious bubbles. Alternatively, if a glass or metal tube, heated by a current of steam, be immersed in the polymer solution for about 10 minutes and then cooled, it will be found, on withdrawal, to have become coated with a layer of jelly.

In more elaborate experiments where the polymer solution was placed between two vertical brass plates, one heated and the other cooled, we have found the concentration of that part of the solution immediately in contact with the hot surface to increase two- or threefold in a short period of time. For example, when a monochlorobenzene solution containing $3 \cdot 1$ per cent of polymethyl methacrylate by weight was confined in the cell for 2 hours, the temperatures of the hot and cold surfaces being approximately $115^{\circ} \mathrm{C}$. and $14^{\circ} \mathrm{C}$. respectively, and the distance between them $1 \mathrm{~cm}$., the jelly adhering to the "hot' wall when the cell was dismantled (after cooling) contained 8.8 per cent solute.

In thermal diffusion it must be supposed that the molecules have a real mean velocity caused by the unsymmetrical field of force arising from the temperature gradient. The resultant force will increase with the size of the molecule and the velocity therefore will remain of the same order of magnitude, or will increase with molecular size, as does the velocity of migration of polymer ions in an electric field. For this reason we consider that the process could more aptiy be called 'thermal migration' rather than thermal diffusion.

$$
\text { G. S. HaRtLey }
$$

Courtaulds, Itd.,

Research Laboratories,

Maidenhead, Berks. Aug. 12.

1 Debye, P., Abstract of Address, Rubber Age, New York, 58, 596 (1946).

\section{Fluorescence Fatigue}

THE fact that substances which fluoresce show a 'fatigue' or 'fading' when exposed continuously to the radiation causing fluor. escence has been noted sporadically. Beese and Marden ${ }^{1}$ investigated the 'fatigue' of several substances, mostly solids, and found that with intense radiation equilibrium was attained in about ten minutes, and that complete recovery was attained in the dark.

Little notice seems to have been taken of fatigue in the biological literature, although it is well known that certain substances such as ribofiavin are light labile. The fading fluorescence of vitamin A, however, has been used in histological investigations and has been studied by Sobotka, Kann and Loewenstein ${ }^{2}$.

In preliminary experiments designed to investigate the physies of the fluorescence of biologically important substances, I have found that fluorescent fatigue is present in all liquids so far investigated: for example, vitamin A, riboflavin, eosin fluorescein, coproporphyrin. Moreover, the curves for the intensity of flurescence apainst time are in some cases strikingly similar to curves of radioactive decay. For example, a dilute watery solution of comparatively pure ribofiavin gives a gives a straight line when the logarithm of the intensity of the fluorescent light is plotted against time. The figure shows a curve for a irradiation.

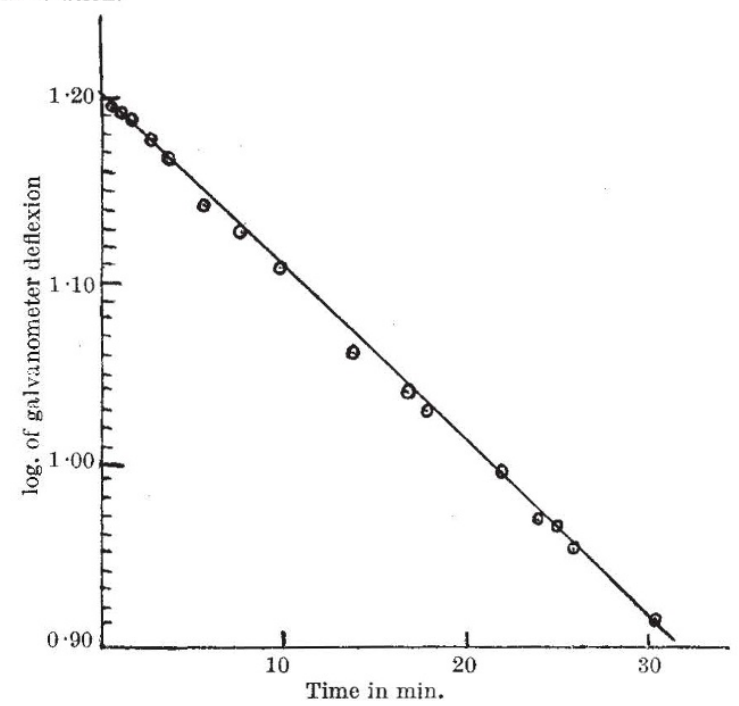

It can be seen that the fluorescence of this sample had a constant rate of decay. The galvanometer readings continued to decrease until the change in a given time interval was of the same order as the fluctuations ordinarily present. Every experiment ended, not with an 'equilibrium' state of the fluorescing material, but with a rate of fading experimental set-up.

Apart from the importance of such data for an understanding of photodynamic action, fluorescent fatigue has an important practical aspect in that measurements of fluorescence intensity have become popular for the assay of biologically important substances. It is, however, apparent that the result of the 'fluorimetry' or 'fluorphotometry' of samples will depend on their previous history as regard
exposure to light.

University of the Witwatersrand,

TIKVAH ALPER

$$
\begin{gathered}
\text { Medical School, } \\
\text { Hospital Street, } \\
\text { Johannesburg. } \\
\text { Aug. } 20 .
\end{gathered}
$$

1 Beese, N. C., and Marden, J. W., J. Opt. Soc. Amer., 32, 317 (1942). Sobotka, H., Kann, S., and Loewenstein, E., J. Amer. Chem. Soc., 65,1959 (1943). 